\title{
A Rare Case of Distal Interphalangeal Chondromatosis in the Middle Finger: Case Report With Review of Literature
}

\author{
LETIZIA SENESI ${ }^{1,2}$, PIER PAOLO PANGRAZI ${ }^{1}$, ANDREA MARCHESINI $^{1}$, FRANCESCO DE FRANCESCO $^{1}$, \\ ANTONIO GIORDANO ${ }^{3}$, ANTONIO GIGANTE ${ }^{2}$ and MICHELE RICCIO ${ }^{1}$ \\ ${ }^{1}$ Department of Reconstructive Surgery and Hand Surgery, Ancona University Hospital, Ancona, Italy; \\ ${ }^{2}$ Clinical Orthopaedics, Department of Clinical and Molecular Science, \\ Polytechnic University of Marche, Ancona, Italy; \\ ${ }^{3}$ Sbarro Institute for Cancer Research and Molecular Medicine, \\ Center for Biotechnology, Temple University, Philadelphia, PA, U.S.A.
}

\begin{abstract}
Primary synovial chondromatosis is a benign condition and consists of cartilaginous metaplasia of the synovium, which leads to the production of intra-articular and periarticular osteocartilaginous bodies. The entity usually involves the synovium of large joints (knee, hip, elbow, and shoulder). It has also been reported in the hand, especially involving interphalangeal or metacarpal joints of digits. The most common symptoms are pain, swelling, loss of range of motion and muscle atrophy. Because of its low prevalence and non-specific symptoms, synovial chondromatosis can present a diagnostic challenge for the hand surgeon and may lead to a delay in treatment. To date, only three cases involving distal interphalangeal joint have been reported. This article describes a rare case of primary articular synovial chondromatosis in the distal interphalangeal joint of a 65 year-old female, giving a deep insight into this pathology, and reviews the literature.
\end{abstract}

Synovial chondromatosis is a benign and proliferative cartilaginous lesion arising from the synovial tissue, tenosynovium, or bursal lining of or near joints (1). Because of its low prevalence and non-specific symptoms, synovial chondromatosis can present diagnostic difficulties for the hand surgeon and may lead to a delay in treatment (2).

This article is freely accessible online.

All Authors contributed equally to this work.

Correspondence to: Francesco De Francesco, Department of Reconstructive Surgery and Hand Surgery, AOU Ospedali Riuniti di Ancona, Via Conca 71, 60126, Ancona, Italy. E-mail: francesco.defrancesco@ospedaliriuniti.marche.it

Key Words: Distal interphalangeal joint, hand, synovial chondromatosis, case report.
Patients may present with pain, swelling, stiffness with/without limitation of motion in the affected joint due to the presence of loose bodies and an active synovium. Examination of the joint may reveal tenderness, crepitus, effusion and palpable nodules, as well as a limited range of motion or locking. It usually involves the synovium of large joints (3) affecting the elbow, the shoulder, the wrist, and the acromioclavicular joints. The rarest location is in the hand (2\% of cases) (4). Location in metacarpophalangeal joints $(2$, 5-9), proximal interphalangeal joints $(2,10-14)$ and thumb (15-18) have been reported in the literature.

The differential diagnosis is not always simple, and includes rheumatoid arthritis, chronic infection (including tuberculosis), trauma (osteochondral fracture), osteochondritis dissecans, osteoarthritis, neuropathic arthritis and gout (9).

We report a case of primary articular synovial chondromatosis in the distal interphalangeal (DIP) joint of a finger of a 65-year-old female.

\section{Case Report}

A 65-year-old female complained of increasing pain, swelling, inflammation in the dorsal aspect of the DIP of the right middle finger, associated with an important reduction of the range of motion and onychodystrophy. No trauma or autoimmune diseases were reported. X-Rays of the hand showed erosive osteoarthritis with classic central erosion of the DIP joint of fingers II, III and IV associated with volar joint dislocation.

The erythrocyte sedimentation rate and C-reactive protein level were both normal.

A new panel of blood analyses was performed in order to exclude autoimmune disease. This included: Rheumatoid factor, C3/C4 complement test, nuclear antibody test, neutrophil cytoplasmatic antibody test, citrullinated protein antibody test, cardiolipine antibody, double-stranded DNA antibodies. All tests gave negative results. 


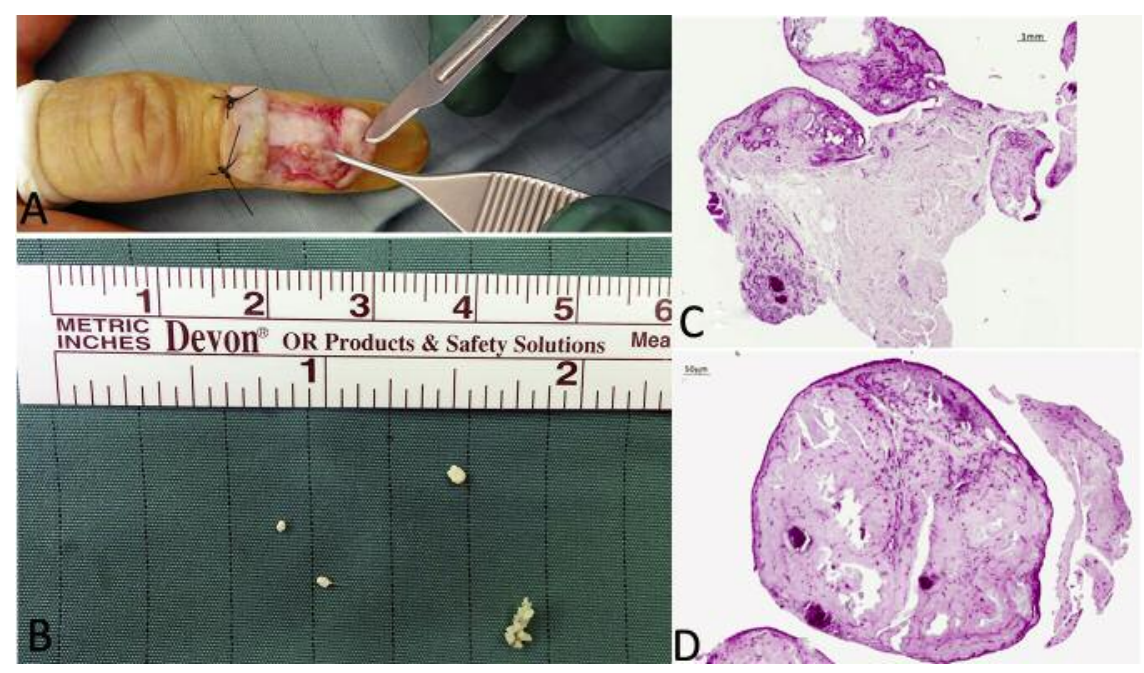

Figure 1. A: Gross view of surgical dissection. B: The loose bodies remove from the distal interphalangeal joint were firm, smooth, yellowish with homogeneous cut surfaces. $C$ and D: Anatomopathological evaluation showed typical round cellular islands of cartilage in fibrous tissue, chondrocytes forming nests of cells, a distinctive aspect of synovial chondromatosis (hematoxyllin-eosin; magnification C: 20x, D:40x). No malignancy was found in this setting.

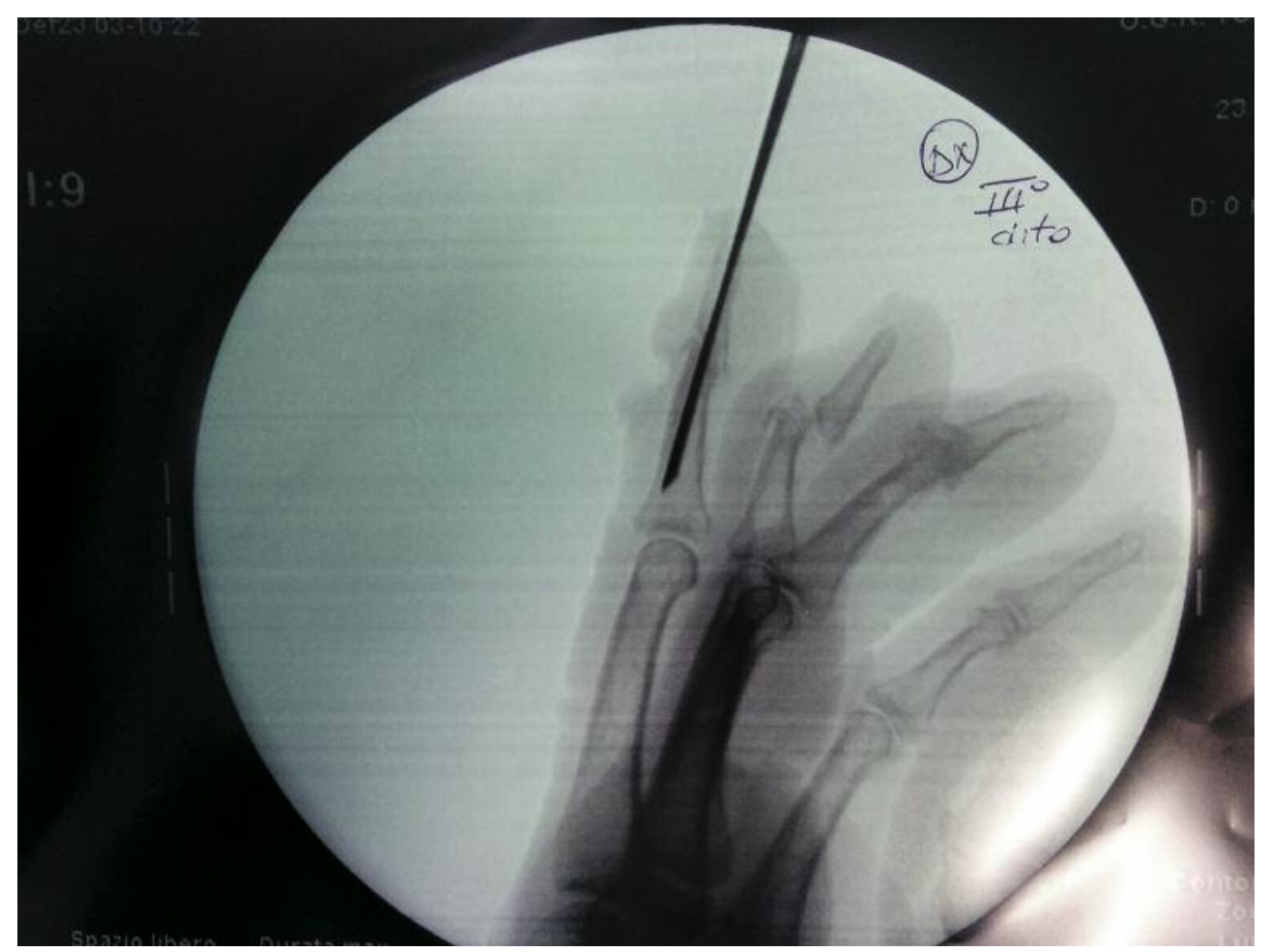

Figure 2. Radiograph performed after the surgical treatment. After removal of loose bodies and the reduction of the volar luxation of the phalanx, arthrodesis of distal interphalangeal joint by two Kirschner wires was carried out.

The patient reported a worsening of functional deficits with increased pain and light touch discomfort. She was therefore advised to undergo surgical exploration, with a diagnoses of erosive osteoarthritis of the hand.
DIP exploration revealed multiple firm smooth yellowish nodules, strongly suggesting a rare case of synovial chondromatosis, which were excised and sent for pathological examination (Figure 1). 

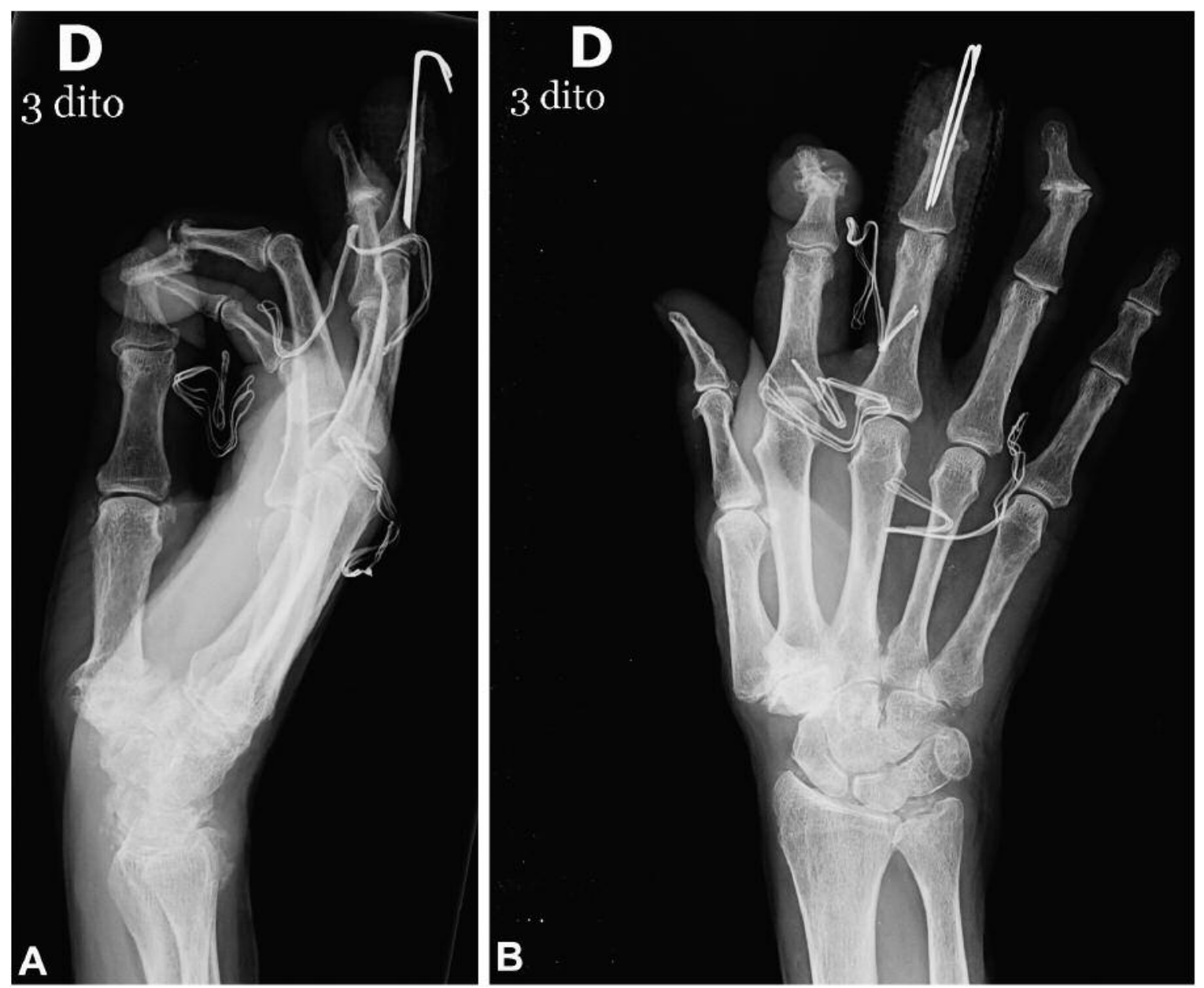

Figure 3. Radiographs performed 30 days after surgery showed initial bone healing.

Therefore, given the state of articular surfaces, in order to maintain finger functionality, surgical arthrodesis of DIP by two Kirschner wires was performed (Figure 2). A splinted cast was applied for 30 days, removed only for physical rehabilitation. Four days after surgery, physical therapy to maintain the range of motion of the proximal interphalangeal joint and metacarpophalangeal joint of the right middle finger was initiated.

At one-month follow-up, X-rays showed a healing callus (Figure 3), and the splinted cast and Kirschner wires were removed.

Histological studies revealed well-circumscribed nodules of cartilage and bone. The cartilage was of moderate cellularity, with varying degrees of ossification, which was consistent with synovial chondromatosis (Figure 1C and D).

Milgram described three separate phases in primary synovial chondromatosis: Stage 1i: Active intrasynovial disease with no free loose bodies; stage 1: transitional lesion

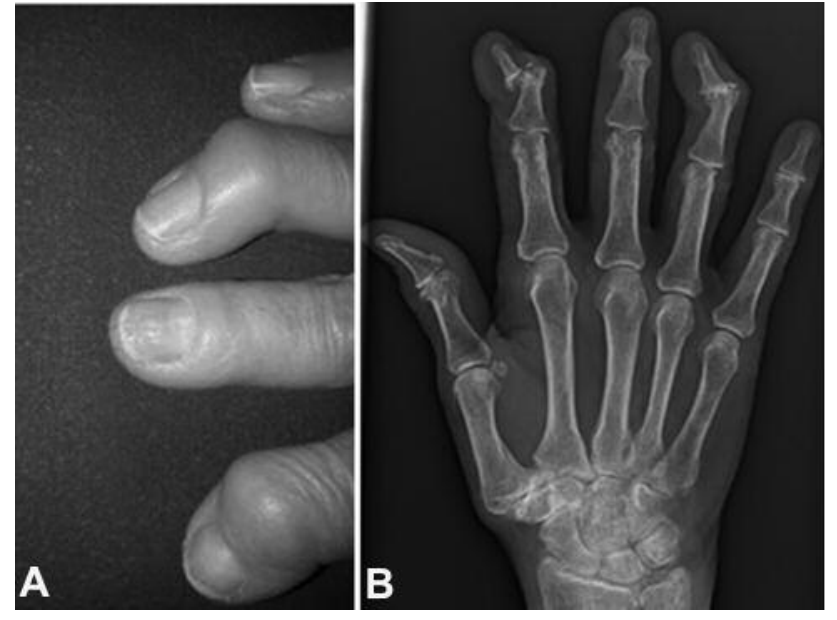

Figure 4. A: Finger presentation 2 years after surgery: there were no signs of inflammation. B: X-Ray performed 2 years after surgery showed no signs of recurrence. 
with osteochondral nodules in the synovial membrane and osteochondral bodies lying free within the joint cavity; stage 3: multiple free osteochondral bodies with now quiescent intrasynovial disease (19). We classified our patient as being in stage 2 by Milgram's classification.

No recurrence was observed after 2-year follow-up (Figure 4).

\section{Discussion}

Primary synovial chondromatosis consists of cartilaginous metaplasia of the synovium, which leads to the production of intra-articular and periarticular osteocartilaginous bodies. Distinction of primary synovial chondromatosis from the more common secondary synovial chondromatosis, which consists of free articular cartilage fragments resulting from degenerative disease or trauma, is not always simple.

As previously stated, this condition rarely involves the upper extremity. Literature reveals that primary articular synovial chondromatosis is exceedingly rare in the DIP joint, with only three cases reported in literature (20-22).

Because of its low prevalence, and inconstant symptoms, diagnosis can often be delayed. The differential diagnosis includes chronic infection, trauma, erosive osteoarthrosis, and autoimmune disease such as systemic lupus erythematosus, rheumatoid arthritis and psoriatic arthritis that were excluded through blood analysis in this case.

Despite the fact that malignant transformation into chondrosarcoma is considered extremely rare, complete excision of the lesion is commonly accepted. Usually pain and other symptoms are relieved by the evacuation of the loose bodies. Synoviectomy is also recommended in literature in order to reduce symptoms (3), even though whether it prevents recurrence is still controversial.

The difficulty in determining the most appropriate treatment in synovial chondromatosis is due to the low incidence of the disease, which precludes carrying out randomized trials. For cases in the DIP joint, there is good reason to proceed to total synoviectomy and arthrodesis immediately $(22,23)$.

Long-term follow-up is indicated for all patients because recurrence is not common but can occur (23). Moreover, malignant transformation into chondrosarcoma is considered extremely rare; in the literature, a relative increased risk of developing chondrosarcoma was reported in a series of 53 cases of synovial chondromatosis (23).

In conclusion, synovial chondromatosis is a rare disease, and its localization in the hand is uncommon. Proper differential diagnosis is crucial in order to recognized and treat it correctly.

\section{Conflicts of Interest}

No pharmaceutical industry or outside support was used for this study.
The Authors report no conflicts of interest. No funding was received for this work.

\section{Authors' Contributions}

PPP and AM perform the operation. LS, FDF and AG wrote the article. AG and MR supervised and correct the article.

\section{References}

1 Ho YY and Choueka J: Synoval chondromatosis of the upper extremity. J Hand Surg 38(4): 804-810, 2013. PMID: 23474166. DOI: $10.1016 /$ j.jhsa.2013.01.041

2 Wong SH-M, Salama S and Thoma A: Synovial chondromatosis of the hand: Three case reports and literature review. Can J Plast Surg 11(1): 47-52, 2003. PMID: 24115851.

3 Maurice H, Crone M and Watt I: Synovial chondromatosis. J Bone Joint Surg 70(5): 807-811, 1988. PMID: 3192585.

4 Kumar N, Mukhopadhaya J and Anand OJ: Synovial chondromatosis of distal radioulna joint with features of lytic lesion in a 29 years male: A case report and review of literature. J Sports Med Doping Stud 6: 174, 2016. DOI: 10.4172/2161-0673.1000174

5 Reed SC and Wright CS: Synovial chondromatosis of the metacarpophalangeal joint: case report and review of the literature. Can J Surg 39(5): 407-409, 1996. PMID: 8857991.

6 Hettiaratchy SP and Nanchahal J: Synovial chondromatosis of the metacarpophalangeal joint. J Hand Surg 27(1): 104-106, 2002. PMID: 11895357. DOI: 10.1054/jhsb.2001.0677

7 Ozcelik IB, Kuvat SV, Mersa B and Pilancı O: Synovial chondromatosis of the metacarpophalangeal joint of the ring finger. Acta Orthop Traumatol Turc 44(4): 337-339, 2010. PMID: 21252612. DOI: 10.3944/AOTT.2010.2324

8 Tominaga A, Takenaka S, Murase T, Hashimoto N, Naka N and Yoshikawa H: Synovial chondromatosis of the metacarpophalangeal joint: A case report and literature review. Hand Surg 17(03): 395-398, 2012. PMID: 23061954. DOI: 10.1142/ S0218810412720409

9 Warne BA, Tigrani DY and Ward CM: Metacarpophalangeal joint synovial osteochondromatosis: A case report. Iowa Orthop J 28: 91-93, 2008. PMID: 19223957.

10 Sano K, Hashimoto T, Kimura K and Ozeki S: Articular synovial chondromatosis of the finger. J Plast Surg Hand Surg 48(5): 347349, 2014. PMID: 23596991. DOI:10.3109/2000656 X.2013. 788308

11 Takami H, Takahashi S and Ando M: Synovial chondromatosis of the proximal interphalangeal joint of a finger. J Hand Surg 19(5): 794-796, 1994. PMID: 7806802. DOI: 10.1016/03635023(94)90185-6

12 Kamath BJ, Bhardwaj P, Shriharsha K and Sharma C: Synovial chondromatosis of the proximal interphalan-geal joint. J Hand Surg 32(1): 108-108, 2007. PMID: 17125894. DOI: 10.1016/ j.jhsb.2006.10.003

13 Spiers JD, Wijeratna MD and Jones JW: Synovial chondromatosis affecting a digital proximal interphalangeal joint. Hand Surg 19(01): 127-129, 2014. PMID: 24641756. DOI: 10.1142/S0218810414720101

14 Kumar A and Thomas AP: Recurrent synovial chondromatosis of the index finger: Case report and literature review. Hand Surg 5(02): 181-183, 2000. PMID: 11301515. 
15 Lewis MM, Marshall JL and Mirra JM: Synovial chondromatosis of the thumb: A case report and review of the literature. JBJS 56(1): 180-183, 1974. PMID: 4812161.

16 Malhotra R, Gaur S, Dave PK and Dinda AK: Synovial chondromatosis of the interphalangeal joint of the thumb: A case report. J Hand Surg 19(3): 460-461,1994. PMID: 8056975. DOI: 10.1016/0363-5023(94)90063-9

17 Gottschalk HP, Newbury R and Wallace CD: Synovial chondromatosis in a child's thumb: a case report and review of the literature. Hand 7(1): 98-102, 2012. PMID: 23450976. DOI:10.1007/s 11552-011-9376-y

18 Muramatsu K, Hashimoto T, Tominaga Y and Taguchi T: Large synovial osteochondromatosis arising from the interphalangeal joint of the thumb: A case report and review of the literature. Anticancer Res 34(2): 1057-1060, 2014. PMID: 24511055.

19 Milgram JW: Synovial chondromatosis a hystopatological study of thirty cases. J Bone Joint Surg Am 59(6): 792-801,1977. PMID: 908703.
20 Harvey FJ and Negrine J: Synovial chondromatosis in the distal interphalangeal joint. J Hand Surg 15(1): 102-105,1990. PMID: 2299147.

21 Craft RO, Smith AA and Duncan SFM: Recurrent synovial chondromatosis of the distal interphalangeal joint: Case report. J Hand Surg 34(2): 301-303, 2009. PMID: 19181231. DOI: 10.1016/j.jhsa.2008.10.020

22 Arnaud JP, Giroud P, Arnaud $M$ and Pecout C: Osteochondromatosis of distal interphalangeal articulation. Ann Chir Main 6(2): 144-146, 1987. PMID: 3426319.

23 Apte SS and Athanasou NA: An immunohistological study of cartilage and synovium in primary synovial chondromatosis. $\mathrm{J}$ Pathol 166(3): 277-281, 1992. DOI: 10.1002/path.1711660310

Received May 1, 2019

Revised May 22, 2019

Accepted May 23, 2019 